\title{
Mastopatia Diabética: Causa Incomum de Doença Inflamatória da Mama
}

\author{
Diabetic Mastopathy: Uncommon Cause of Inflammatory Disease of the Breast
}

Juvenal Mottola Jr., Fábio Martins Laginha Cídia Mazzoccato, Anastásio Berretini Jr., Maria do Carmo Assunção

\begin{abstract}
RESUM0
Objetivo: estudar a associação entre diabete melito insulino-dependente de longa evolução com mau controle glicêmico e lesões inflamatórias da mama que podem, por vezes, simular carcinoma inflamatório.

Métodos: no período de janeiro de 1998 a dezembro de 2001 foram estudadas, retrospectivamente, 18 pacientes em serviço de referência de mastologia que apresentavam lesão inflamatória da mama com ou sem tumoração palpável. As mesmas foram submetidas à dosagem sérica de glicose e hemoglobina glicosilada, bem como exames de imagem e análise histológica da glândula mamária, e tiveram como diagnóstico mastopatia diabética.

Resultados: a média etária das pacientes foi de 50,2 anos e todas eram portadoras de diabete melito insulino-dependente, com tempo médio de evolução da doença de 14,9 anos. Todas apresentaram mau controle glicêmico, com glicemia sérica média de 329,6 mg/dL e hemoglobina glicosilada média de 9,7\%. A dose média de insulina NPH utilizada ao dia era de 37,2 unidades. As pacientes foram submetidas a tratamento clínico com antibioticoterapia e controle dos níveis glicêmicos com insulina NPH e simples e tiveram resolução do quadro em aproximadamente cinco semanas.

Conclusão: os profissionais envolvidos com cuidados à saúde da mulher precisam estar cientes desta patologia inflamatória das mamas e de seu caráter benigno, para evitarem-se condutas desnecessárias, muitas vezes prejudiciais à paciente.
\end{abstract}

PALAVRAS-CHAVE: Mama: doença benigna. Mama: infecções. Mama: câncer. Diabete melito.

\section{Introdução}

Descrita pela primeira vez em 1984 por Soler e Khardori ${ }^{1}$, a mastopatia diabética é forma rara de mastite, que ocorre em pacientes diabéticas insulino-dependentes de longa duração com mau controle glicêmico. Apresenta-se como lesão inflamatória da mama, acometendo, principalmente, mulheres na pré-menopausa. Representa menos de $1 \%$ das doenças benignas da mama, porém, pode acometer até $13 \%$ das pacientes diabéticas em alguma época durante o curso de sua doença ${ }^{2}$. Sua patogênese não é totalmente conhecida; acre-

Centro de Referência da Saúde da Mulher, Hospital Pérola Byington, SP

Correspondência:

Juvenal Mottola Jr.

R. Dr. Zuquim 449 cj. 51/52

02035-010 - São Paulo - SP

e-mail: jmottola@uol.com.br dita-se que seja devida a uma reação auto-imune induzida pela hiperglicemia, com expansão da matriz e formação de antígeno na glândula mamária, com acúmulo de linfócitos e com a proliferação de miofibroblastos epitelióides ${ }^{3,4}$. Apresenta-se, geralmente, como tumoração palpável uni ou bilateral, com características inflamatórias, recorrente e inicialmente indolor, podendo tornarse dolorosa com a evolução do quadro clínico. O principal diagnóstico diferencial deve ser feito com carcinoma lobular invasivo e carcinoma inflamatório, os quais a mastopatia diabética pode simular clinicamente. O exame histopatológico é fundamental para se estabelecer esta diferenciação. Outras patologias mamárias devem ser excluídas, como: mastite de células plasmáticas, fibrose mamária involucional, doenças fibrocísticas e linfoma não-Hodgkin primário da mama ${ }^{5,6}$. O tratamento baseia-se em controlar os níveis 
glicêmicos, o processo inflamatório e, eventualmente, infecções secundárias, com esquema de antibióticos com espectro para microrganismos anaeróbios e gram-positivos. É desnecessário qualquer outro procedimento cirúrgico além da biópsia para exclusão de malignidade. O presente estudo teve como objetivo estudar a associação entre diabete melito insulino-dependente de longa duração com mau controle glicêmico e lesões inflamatórias da glândula mamária que podem, por vezes, simular carcinoma inflamatório.

\section{Pacientes e Métodos}

Foram analisadas, retrospectivamente, as informações sobre 18 pacientes com diagnóstico de mastite associada ao diabete melito atendidas no serviço de referência em mastologia do Centro de Referência da Saúde da mulher, Hospital Pérola Byington, no período compreendido entre janeiro de 1998 e dezembro de 2001. A inclusão das pacientes no estudo foi estabelecida de acordo com o protocolo de assistência às pacientes com processo inflamatório das mamas e aprovado pela Comissão de Ética do hospital, com assinatura do consentimento pós-informado de assistência institucional.

O diagnóstico de mastopatia diabética foi estabelecido pela história clínica, com a queixa de processo inflamatório das mamas de aparecimento recente, por vezes recorrente, associado à história de diabete melito insulino-dependente de longa evolução com mau controle glicêmico, e pelo exame físico revelando hiperemia, aumento do volume mamário com tumor palpável ou não, aumento da temperatura local, edema de pele e até epidermólise e abscessos, caracterizando, assim, o grupo de pacientes incluídas neste estudo. As pacientes foram submetidas ao exame físico completo e complementar para excluir outras causas de mastites, como a tuberculose, sífilis, mastites periareolares recidivantes complicadas por fístulas e abscessos mamários e mastites pós-necrose gordurosa por trauma da glândula mamária.

Os exames subsidiários foram a ultra-sonografia, para pesquisa de abscessos intra ou retroglandulares e para avaliação de nódulos mamários coexistentes. O estudo mamográfico foi realizado, principalmente, para avaliação da glândula mamária contra-lateral e da acometida, após a resolução do processo agudo, para se excluir patologia neoplásica. Os achados aos exames de imagem não foram específicos para o diagnóstico de mastopatia diabética. Os exames de glicemia de jejum sérica pelo método calorimétrico e da dosa- gem de hemoglobina glicosilada no sangue total pelo método de cromatografia líquida de alta pressão (HLPC) foram considerados normais para a instituição quando os valores foram de 75 a 110 $\mathrm{mg} / \mathrm{dL}$ e 3,6 a 5,3\%, respectivamente. A biópsia percutânea por agulha grossa ("core biopsy") da mama afetada, realizada durante o período de tratamento, foi o método de escolha para o estudo histopatológico da lesão.

\section{Resultados}

Um total de 18 pacientes com diagnóstico de mastopatia diabética foi analisado e constituiu a casuística do presente estudo. A idade destas pacientes variou entre 33 e 63 anos (média de 50,2 anos) e todas eram portadoras de diabete melito insulino-dependentes com tempo médio de evolução da doença de 14,9 anos (10 a 22 anos). Todas, sem exceção, apresentavam mau controle glicêmico, com glicemia sérica média de 329,6 $\mathrm{mg} / \mathrm{dL}$ (245 a $485 \mathrm{mg} / \mathrm{dL}$ ) e hemoglobina glicosilada média de $9,7 \%$ (8 a 12\%). A dose média de insulina NPH utilizada ao dia era de 37,2 unidades (25 a $60 \mathrm{U}$ ) e nenhuma delas encontrava-se em uso de insulina simples. O tempo médio desde o aparecimento dos sintomas até a procura por atendimento especializado foi de cinco meses (variando de 2 a 8 meses). Clinicamente, todas as pacientes apresentavam tumoração em uma das mamas; em 5 pacientes $(27,7 \%)$ ocorreu hiperemia da mama acometida e edema de pele adjacente ao tumor, simulando carcinoma inflamatório. Observou-se em uma paciente epidermólise com exsudato purulento. As demais pacientes apresentavam características clínicas de processo inflamatório inespecífico, com hiperemia e abscesso mamário (Figura 1). Foi realizada mamografia após a resolução do processo inflamatório em 16 pacientes. Em todas se evidenciou parênquima mamário assimétrico. Com uso das técnicas de compressão focal houve atenuação e modificação da sua forma. Houve perda de seguimento radiológico em duas pacientes, que não retornaram ao ambulatório na data agendada para revisão. Ao exame ultra-sonográfico, os achados foram de área hipoecóica mal delimitada, com sombra acústica posterior, que o ultra-sonografista diagnosticou como achados inespecíficos em 11 pacientes $(61,1 \%)$. As demais 7 pacientes não foram submetidas à ultra-sonografia. Seis pacientes foram submetidas à drenagem cirúrgica com biópsia do tecido perilesional $(33,3 \%)$. Em outras nove pacientes (50\%) foram realizadas biópsias percutâneas 
(“core-biopsy") e em apenas três pacientes foi realizada punção aspirativa com agulha fina (PAAF). Entre estas o material foi insuficiente em todas, realizando-se então a biópsia por fragmento. Os achados ao exame histopatológico incluíram lobulite e ductite linfocítica, perivasculite, tecido fibroso denso envolvendo os lóbulos, espessamento da membrana basal, esclerose do estroma interlobular, atrofia ductal e, comum a todos, microabscessos mamários (Figura 2). A conduta terapêutica envolveu avaliação clínica objetivando o controle glicêmico com dieta adequada e ajuste das doses de insulina NPH e simples até se obter niveis de glicemia inferiores a $140 \mathrm{mg} / \mathrm{dL}$. O período médio de resolução de tratamento foi de 36,8 dias, variando entre 28 a 47 dias. Houve seguimento completo em apenas 6 pacientes $(33,3 \%)$ e estas não apresentaram recorrência nestes 24 meses de seguimento.

Figura 1 - Diferentes formas de apresentações clínicas da mastopatia diabética, por vezes simulando carcinoma inflamatório.

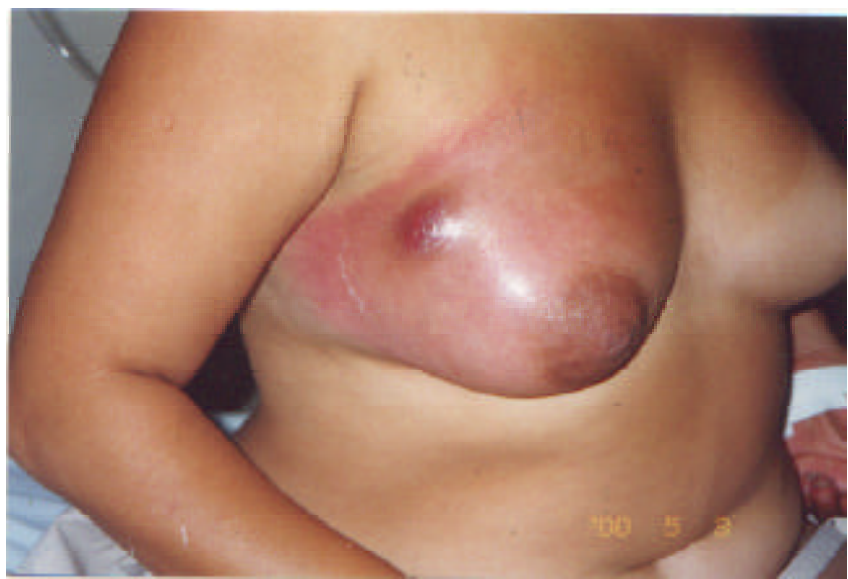

Figura 1.A - Hiperemia da mama com edema da pele adjacente, simulando carcinoma inflamatório.

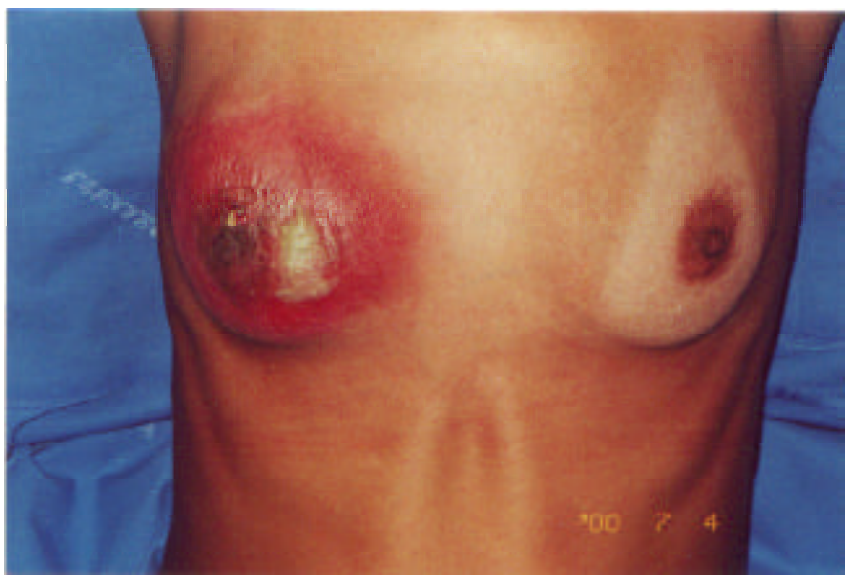

Figura 1.B - Epidermólise da pele sobre a área abscedada.

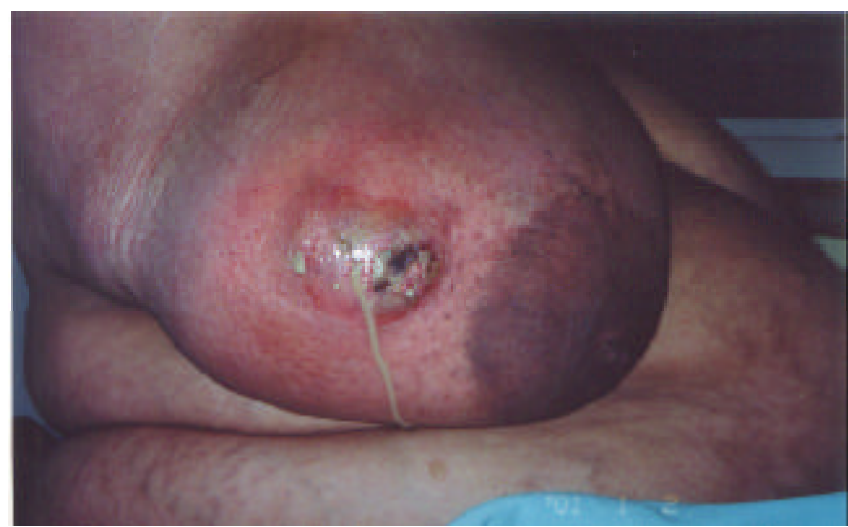

Figura 1.C - Hiperemia, epidermólise da pele e exsudato purulento.

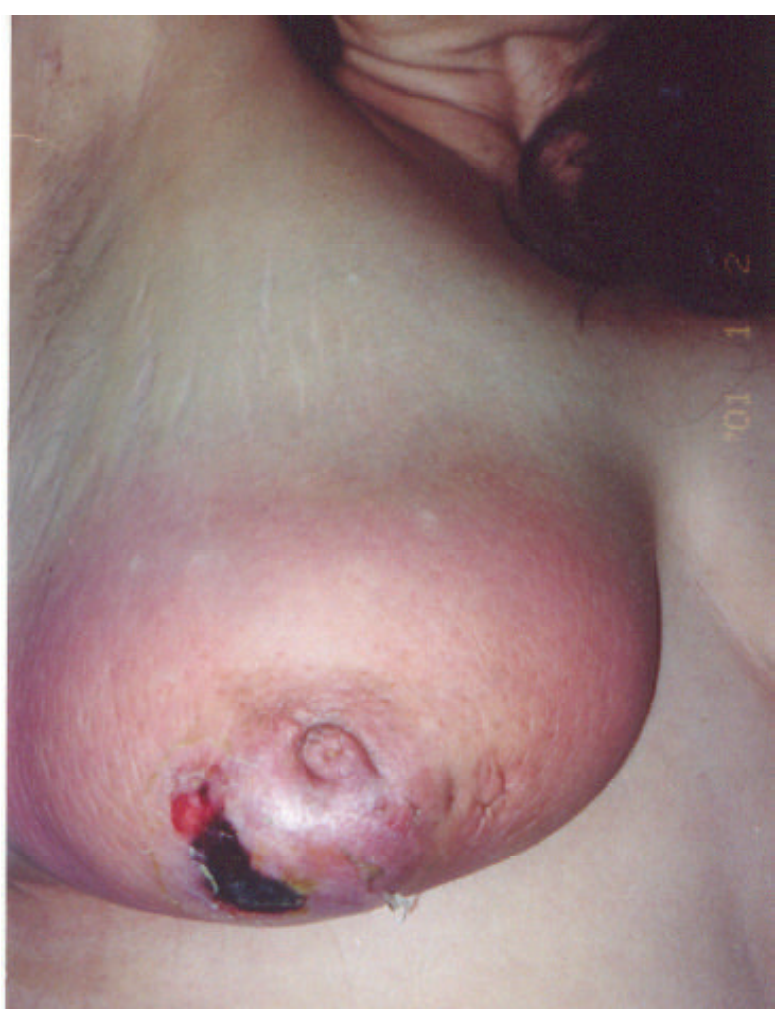

Figura 1.D - Epidermólise, hiperemia e perda de tecido na região da pele ulcerada.

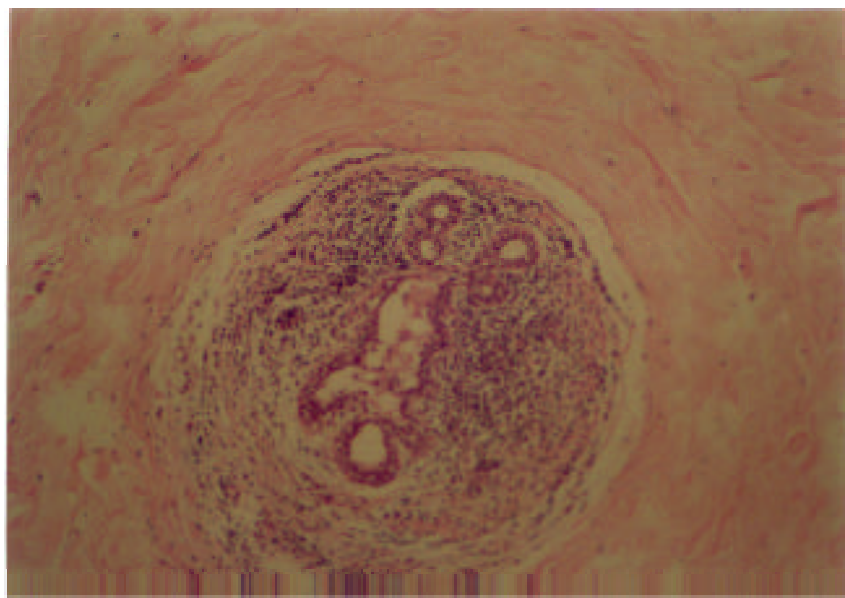

Figura 2 - Aspecto histológico da mastopatia diabética apresentando lobulite, ductite linfocítica e microabscessos. HE 10X. 


\section{Discussão}

Desde a primeira publicação em 1984 por Soler e Khardori ${ }^{1}$ que relataram doze casos de pacientes que apresentavam associação de doença fibrosa da mama, tiroidite e artropatia das mãos com diabete melito tipo I, um total de 110 casos de mastite linfocítica associada à diabete melito foi relatado na literatura até o ano de $1997^{2}$. Embora não existam dados epidemiológicos que forneçam informação precisa a respeito da freqüência real da mastopatia diabética, trata-se de uma enfermidade rara, sendo sua freqüência estimada em 1:1694 mulheres diabéticas ${ }^{3}$. Representa menos de $1 \%$ das doenças benignas da mama, porém, é achado mais freqüente em pacientes diabéticas insulino-dependentes com mau controle glicêmico, acometendo 13\% destas ${ }^{2}$. No ano de 1999, em nosso ambulatório de referência para doenças mamárias, foram atendidas 5000 novas pacientes. Destas, 4470 pacientes apresentavam doenças benignas da mama, dentre as quais, 350 casos de processos inflamatórios, entre eles, seis casos de mastopatia diabética.

Embora vários mecanismos tenham sido propostos para explicar a patogênese da mastopatia diabética, nenhum foi cientificamente comprovado. O papel da auto-imunidade foi primeiramente sugerido por Soler e Khardori ${ }^{1}$ e a associação foi confirmada por Tomaszewski e et al. ${ }^{3}$ e Tanière et al. ${ }^{7}$, que observaram ligação entre diabete, doenças da tiróide e anormalidades do tecido conectivo, como lúpus eritematoso sistêmico. Esta associação seria explicada por uma reação imune ao acúmulo de matriz anormal no tecido mamário induzida pela hiperglicemia. Seidman et al. ${ }^{8}$ propuseram que a insulina exógena poderia levar ao desenvolvimento de mastopatia diabética devido a uma reação inflamatória ou imunológica à insulina, ao seu veículo ou à contaminação deste.

Não há um consenso que estabeleça os critérios para o diagnóstico inequívoco da mastopatia diabética. A história da paciente, os achados ao exame físico e radiológico e a análise de diagnóstico citológico por biópsia aspirativa com agulha fina (BAAF) e histológico, quer seja por biópsia cirúrgica ou "core biopsy" levam ao diagnóstico desta doença ${ }^{5}$.

Clinicamente apresenta-se, geralmente, como tumor mamário palpável em pacientes diabéticas tipo I de longa duração com mau controle glicêmico, podendo raramente acometer diabéticas tipo $\mathrm{II}^{3}$ que após o diagnóstico passaram a necessitar de terapia com insulina. Geralmente, a tumoração é diagnosticada pela própria paciente e tem consistência endurecida, com bordas irregu- lares, móvel e indolor. Pode manifestar-se como massa unilateral, bilateral ou lesão não palpável, tendo propensão pela região subareolar ${ }^{4,7}$. O diâmetro das lesões descritas na literatura pode variar entre $5 \mathrm{~mm}$ e $6 \mathrm{~cm}^{4}$. Taxas de recorrência de até $32 \%$ foram relatadas ${ }^{8}$ e o reconhecimento desta característica é fundamental para evitar biópsias mamárias repetidas. Apesar de acometer, predominantemente, pacientes do sexo feminino, há descrição na literatura de casos de mastopatia diabética em homens, ressaltando a importância da vigilância na população de homens diabéticos ${ }^{9,10}$.

Os achados radiológicos, assim como os clínicos, não são específicos e podem causar confusão com carcinoma mamário. A área comprometida manifesta-se à mamografia como um tecido extremamente radiodenso e homogêneo, que não é específico da doença, já que é descrito em $27 \%$ dos exames mamográficos em mulheres menopausadas não diabéticas ${ }^{5,11}$. Ao exame ultra-sonográfico pode apresentar-se como um nódulo hipoecóico, irregular. com marcada sombra acústica posterior, simulando uma lesão maligna ${ }^{11}$.

O processo diagnóstico da mastopatia diabética pela citologia obtida por PAAF é prejudicado devido à característica consistente do tecido fibroso, que impede a adequada amostragem citológica. Logan e Hoffman ${ }^{12}$ demonstraram que em $50 \%$ dos casos por eles avaliados o aspirado continha material celular insuficiente para avaliação, tornando necessária amostragem histológica. Como passo seguinte, o diagnóstico por biópsia percutânea com agulha grossa pode substituir com precisão a biópsia cirúrgica ${ }^{13}$. Andrews-Tang et al. ${ }^{13}$ relataram falha diagnóstica no exame citológico devido ao número de casos descritos como material insuficiente para diagnóstico, enquanto que em biópsia por fragmento por agulha grossa ("core biopsy") dirigida pelo exame ultra-sonográfico, o diagnóstico foi conclusivo em 10 exames de 11 realizados ${ }^{6}$. Os achados histológicos incluem fibrose estromal densa associada à infiltração linfocítica ductal e lobular e vasculite linfocítica, predominantemente de células B. Tomaszewsky et al. ${ }^{3}$ relataram o achado de células epitelióides peculiares embebidas no estroma fibroso denso, que denominaram "fibroblastos epitelióides". Postulou-se que este achado era específico de biópsias de mama de pacientes diabéticas, porém, posteriormente foi encontrado em amostras de biópsias de carcinoma e de tumor de células granulares em pacientes não-diabéticas, não sendo assim elemento específico para esta população de pacientes, mas sim um dado sugestivo de alterações mamárias em pacientes diabéticas ${ }^{2}$.

O tratamento consiste em uma adequada avaliação clínica, objetivando o controle glicêmico com dieta adequada e ajuste das doses de insu- 
lina NPH e simples até obter níveis glicêmicos abaixo de $140 \mathrm{mg} / \mathrm{dL}$. Deve-se associar antibioticoterapia em casos de infecção secundária, com espectro para microorganismos gram positivos e anaeróbios, como cefalosporinas de primeira geração na dose de $2 \mathrm{~g} /$ dia e clindamicina na dose de 1800 mg/dia. Aguarda-se o resultado da cultura da secreção, sempre que houver condições para realizá-la, modificando o esquema de antibióticos se o germe for resistente ao citado acima ${ }^{8}$.

O principal diagnóstico diferencial deve ser feito com carcinoma lobular invasivo e carcinoma inflamatório ${ }^{6,14}$. Outras patologias mamárias devem ser excluídas, como mastite de células plasmáticas, fibrose mamária involucional, doenças fibrocísticas e linfoma não-Hodgkin primário da mama ${ }^{5,15}$.

Somente a integração entre a história clínica, achados ao exame físico e radiológico, citologia e histologia pode levar ao diagnóstico acurado desta doença benigna. Acreditamos que os profissionais envolvidos nos cuidados à saúde da mulher precisam estar atentos à associação entre diabete melito de longa duração e processos inflamatórios da mama, para adequadamente tratá-la e evitarse assim condutas desnecessárias.

\section{ABSTRACT}

Purpose: to study the association between long-standing type 1 diabetes with bad glycemic control and breast inflammatory lesions which can simulate inflammatory carcinoma.

Patients and Methods: eighteen patients were studied, retrospectively, in a mastology reference center from January 1998 to December 2001, presenting with breast inflammatory lesion with or without palpable mass. They were submitted to serum glucose and glycosylated hemoglobin determination, as well as image examination and histopathologic analysis, and diabetic mastopathy was diagnosed.

Results: the patients' average age was 50.2 years, and all had insulin-dependent diabetes mellitus, with average disease time of 14.9 years. All patients, with no exception, had a bad glycemic control; the average blood glucose was $329.6 \mathrm{mg} / \mathrm{dL}$ and the glycosilated hemoglobin average was 9.7\%. NPH insulin dose being applied per day was 37.2 units. Patients underwent a clinical treatment with antibiotics and control of the glycemic levels with NPH insulin and had resolution of the symptoms in about five weeks.

Conclusion: the professionals involved in women health care must be aware of this inflammatory pathology of the breast and its benign characteristics to avoid unnecessary procedures sometimes with patient injury.

KEY WORDS: Breast: benign disease. Breast cancer. Breast infections. Diabetes mellitus.

\section{Referências}

1. Soler NG, Khardori R. Fibrous disease of the breast, thyroiditis and cheiroarthropathy in type I diabetes mellitus. Lancet 1984; 1:193-5.

2. Hunfeld KP, Bässler R. Lymphocytic mastitis and fibrosis of the breast in long-standing insulindependent diabetics. A histopathologic study on diabetic mastopathy and report of ten cases. Gen Diagn Pathol 1997; 143:49-58.

3. Tomaszewski JE, Brooks JS, Hicks D, Livolsi VA. Diabetic mastopathy: a distinctive clinicopathologic entity. Hum Pathol 1992; 23:780-6.

4. Ely KA, Tse G, Simpson JF, Clarfeld R, Page DL. Diabetic mastopathy. A clinicopathologic review. Am J Clin Pathol 2000; 113:541-5.

5. Boullu S, Andrac L, Piana L, Darmon P, Dutour A, Oliver C. Diabetic mastopathy, complication of type 1 diabetes mellitus: report of two cases and a review of the literature. Diabetes Metabol 1998; 24:448-54.

6. Kribi L, Chelli M, Sellami D, et al. La mastopathie fibreuse diabétique. J Radiol 1999; 80:1579-81.

7. Tanière P, Poulard G, Frappart L, et al. Mastopathie diabétique avec fibroblastes épithélioïdes: piège diagnostique avec un carcinome lobulaire infiltrant du sein. A propos de deux cas. Ann Pathol 1996; 16:33-6.

8. Seidman JD, Schnaper LA, Phillips LE. Mastopathy in insulin-requiring diabetes mellitus. Hum Pathol 1994; 25:819-24.

9. Hunfeld KP, Bässler R, Kronsbein H. "Diabetic mastopathy" in the male breast: a special type of gynecomastia. A comparative study of lymphocytic mastitis and gynecomastia. Pathol Res Pract 1997; 193:197-205.

10.Cavazza A, Nigrisoli E, Tinterri C, Pedrazzoli C, De Marco L, Gardini G. Mastopatia diabetica maschile: descrizione di un caso. Pathologica 1997; 89:159-62.

11.Feder JM, de Paredes ES, Hogge JP, Wilken JJ. Unusual breast lesions: radiologic-pathologic correlation. Radiographics 1999; 19 Spec No:S11-26.

12.Logan WW, Hoffman NY. Diabetic fibrous breast disease. Radiology 1989; 172:667-70.

13.Andrews-Tang D, Diamond AB, Rogers L, Butler D. Diabetic mastopathy: adjunctive use of ultrasound and utility of core biopsy in diagnosis. Breast $\mathrm{J} 2000$; 6:183-8.

14.Rode S, Favre C, Thivolet C. Diabetic mastopathy. A frequent source of confusion with lobular breast carcinoma. Diabetes Care 1998; 21:332.

15.Pouchot J, Foucher E, Lino M, Barge J, Vinceneux P. Granulomatous mastitis: an uncommon cause of breast abscess. Arch Intern Med 2001; 161:611-2. 